\title{
Endovenöse Laserablation bei Stammvarikosis der Vena saphena magna (VSM) und Vena saphena parva (VSP) sowie bei VSM- Krossenrezidiven (KR) mit neuer radiär abstrahlender Laserfaser - 3 Monatsergebnisse einer prospektiven single-centre-Studie
} Endovenous laser ablation of the incompetent great saphenous
vein (GSV), small saphenous vein (SSV), and GSV recurrences with
stump (SR) using a new $1470 \mathrm{~nm}$ laser and fiber, 3-month results
from a prospective single-centre study

Autoren

Martin Kiderlen*, Nina Schäffer*, Lisa Schuler, Athanasios Theodoridis, Iris Weingard, Nicola Kriechenbauer, Karsten Hartmann

Institute

Venenzentrum Freiburg

\section{Schlüsselwörter}

Venenablation, Krossenrezidiv, Vena saphena magna, Vena saphena parva, Laser, Varikosis

Key words

endovenous laser treatment, great saphenous vein, small saphenous vein, great saphenous vein recurrences, laser, varicose veins

eingereicht 07.07 .2018

akzeptiert $\quad 06.02 .2019$

Bibliografie

DOI https://doi.org/10.1055/a-0853-3256

Phlebologie 2019; 48: 177-181

(c) Georg Thieme Verlag KG Stuttgart · New York

ISSN 0939-978X

Korrespondenzadresse

Dr. Martin Kiderlen

Venenzentrum Freiburg

Zähringer Str. 14, 79108 Freiburg

E-Mail: martin.kiderlen@gmx.de

Englische Version unter:

https://doi.org/10.1055/a-0853-3256

\section{ZUSAMMENFASSUNG}

Einleitung Die thermische Venenablation varikös veränderter Stammvenen der unteren Extremität ist in Deutschland aufgrund der guten Wirksamkeit, Anwendungssicherheit und

* geteilte Erstautorenschaft hoher Patientenzufriedenheit eine anerkannte und häufig durchgeführte Behandlungsmöglichkeit. Zielsetzung der prospektiven Anwendungsbeobachtung (AWB) war die Untersuchung des Operationserfolges, der Patientenzufriedenheit und des Auftretens von Nebenwirkungen bei endovenöser Behandlung der varikös veränderten Vena saphena magna (VSM), Vena saphena parva (VSP) sowie bei Krossenrezidiven (KR) unter Verwendung der ETQ $360^{\circ}$ FUSED Fiber ${ }^{\mathrm{TM}}$.

Material und Methoden Insgesamt wurden 162 varikös veränderte Venen (VSM $n=76$, VSP $n=65, K R n=25)$ bei 144 Patienten behandelt und in die AWB eingeschlossen. Ein duplexsonographisches Follow-up erfolgte nach 10-14 Tagen sowie 3 Monaten. Die ETQ $360^{\circ}$ FUSED Fiber ${ }^{\mathrm{TM}}$ hat eine Wellenlänge von $1470 \mathrm{~nm}$ bei einem beam angle von $60^{\circ}$. Für die endovenöse Therapie der VSM und von KR wurde eine Faser mit einem Faserkerndurchmesser von $600 \mu \mathrm{m}$ bei einem Spitzendurchmesser von 1,8 mm und 8 Watt Behandlungsenergie eingesetzt. Zur Behandlung der VSP wurde ein Faserkerndurchmesser von $400 \mu \mathrm{m}$ bei einem Spitzendurchmesser von 1,3 mm und 6 Watt Behandlungsenergie gewählt.

Ergebnisse Alle thermisch behandelten VSM $(n=6)$ und VSP $(n=65)$ waren bei Visite 2 und Visite 3 vollständig verschlossen. In der KR Gruppe $(\mathrm{n}=25)$ zeigten sich bei Visite 2 Verschlussraten von $96 \%$ und Teilverschlussraten von $4 \%$. Bei Visite 3 konnten noch Verschlussraten von $80 \%$ und Teilverschlussraten von $16 \%$ nachgewiesen werden. In $4 \%$ der Fälle kam es zu einer vollständigen Rekanalisierung. Tiefe Beinvenenthrombosen (TVT) oder Lungenembolien (LE) wurde in keiner der Kohorten diagnostiziert. 1,4\% der an der VSM behandelten Patienten gaben bei Visite 3 Sensibilitätsstörungen im Behandlungsareal an, in der VSP Gruppe waren es 6,15\%. Patienten der KR Kohorte gaben keinerlei Einschränkungen bezüglich der Sensibilität an. Die Patientenzufriedenheit war in allen Gruppen sehr hoch, in allen 3 Gruppen verbesserte sich der Venous Clinical Severity Score (VSCC) signifikant. 
Zusammenfassung Die Behandlung der VSM, VSP und von KR mit der ETQ $360^{\circ}$ FUSED Fiber ${ }^{\mathrm{TM}}$ ist effizient, komplikationsarm und mit einer hohen Patientenzufriedenheit assoziiert.

\section{ABSTRACT}

Introduction The thermal ablation of varicose saphenous veins of the lower extremities is a well-accepted treatment option in Germany due to its high efficacy, application safety, and high patient satisfaction. The objective of the prospective observational study (POS) was to investigate surgical success, patient satisfaction, and the occurrence of side effects in the treatment of the great saphenous vein (GSV), small saphenous vein (SSV), great saphenous vein recurrences (SR) using the ENDOTEQ $360^{\circ}$ FUSED Fiber ${ }^{\mathrm{TM}}$.

Material and Methods The ENDOTEQ $360^{\circ}$ FUSED Fiber ${ }^{\mathrm{TM}}$, operates at a wavelength of $1470 \mathrm{~nm}$ with a beam angle of $60^{\circ}$. A total number of 162 varicose veins (GSV $n=76, S S V n=65$, SR $\mathrm{n}=25$ ) in 144 patients were included in the POS and followed up over a period of 3 months. For the treatment of GSV and SR, a fibre with a core diameter of $600 \mu \mathrm{m}$, tip diameter of $1.8 \mathrm{~mm}$ and $8 \mathrm{~W}$ of energy was used. For the treatment of the SSV, a fibre with a core diameter of $400 \mu \mathrm{m}$, a tip diameter of $1.3 \mathrm{~mm}$ and $6 \mathrm{~W}$ of energy was selected.

Results At the follow-up visit 10-14 days post surgery (visit 2 ) and 3 months post surgery (visit 3), all thermally treated GSVs $(n=76)$ and SSVs $(n=65)$ were occluded. In the SR group $(n=25)$, at visit 2 follow-up, $96 \%$ of SRs remained occluded, and $4 \%$ were partially occluded. Visit 3 showed a closure rate of $80 \%$, a partial closure rate of $16 \%$ and complete recanalisation in $4 \%$ of treated veins. Deep vein thrombosis (DVT) or pulmonary embolism (PE) did not occur in any group. Sensitivity disorders were reported in $1.4 \%$ of the GSV group at visit 3, 6.15\% in the SSV group and none in the SR group. Patient satisfaction was very high in all groups, VSCC improved significantly in all three groups.

Summary Treatment of GSV, SSV, and SR with the ENDOTEQ $360^{\circ}$ FUSED Fiber ${ }^{\mathrm{TM}}$ laser system is efficient, has low complication rates and high patient satisfaction.

\section{Einleitung}

Die thermische Behandlung varikös veränderter Stammvenen der unteren Extremität wird in Deutschland aufgrund der guten Wirksamkeit, Anwendungssicherheit und der hohen Patientenzufriedenheit häufig durchgeführt. Die am häufigsten durchgeführten Verfahren sind die Radiofrequenzablation (RFA) sowie die Laserablation (EVLA). Die Laserablation wurde erstmals 1998 durchgeführt und entwickelt sich seither weiter. Zunächst wurde eine Barefiberfaser (Wellenlänge 810-980 nm) eingesetzt. Eine Verbesserung der Wirksamkeit und eine Reduktion von Nebenwirkungen konnte durch Einführung von Radiallasern mit einer Wellenlänge von 1320-1550 nm erreicht werden. Vorteil von größeren Wellenlängen ist das Absorptionsmaximum in wasserhaltigen Strukturen wie dem Endothel. So werden die Effektivität der Laserenergie in der Gefäßwand erhöht und dadurch höhere Verschlussraten erreicht $[2,4]$.

\section{Material und Methoden}

Die meisten auf dem Markt vorhandenen Radialfasern haben einen Abstrahlwinkel von ca. $80^{\circ}$ mit gezielter Energieabgabe auf die Gefäßwand. Studien zur Wirksamkeit und Sicherheit der Methode sind vorhanden $[1,4,5,8]$. Die ETQ $360^{\circ}$ FUSED Fiber ${ }^{\mathrm{TM}}$ ist eine Radialfaser mit einer Wellenlänge von $1470 \mathrm{~nm}$ bei einem Abstrahlwinkel von $60^{\circ}$. Die Interaktion zwischen Gewebe und der Sonde findet durch den geringeren Abstrahlwinkel an der vorderen Spitze der Sonde statt. So sollten eine Karbonisierung der Sonde sowie Verklebungen mit dem Gewebe verringert werden. Durch den geringeren Abstrahlwinkel sollten außerdem Nebenwirkungen wie z. B. Sensibilitätsstörungen vermindert und gleichzeitig erhöhte Verschlussratewerden erzielt werden. Zur Wirksamkeit und Sicherheit dieser Laserfaser gibt es bisher keine Studiendaten.

Im Zeitraum von September 2016 bis April 2017 haben wir im Venenzentrum Freiburg eine prospektive Anwendungsbeobach- tung (AWB) nach Laserablation der Vena saphena magna (VSM), Vena saphena parva (VSP) und von Krossenrezidiven (KR) bei lang belassenem Krossenstumpf mit der neuen Radiallaserfaser ETQ $360^{\circ}$ FUSED Fiber ${ }^{\mathrm{TM}}$ der Firma Endoteq durchgeführt.

Zielsetzung der prospektiven AWB waren neben der Untersuchung des Operationserfolges sowie der Nebenwirkungsraten auch die Ermittlung der Patientenzufriedenheit anhand einer Schulnotenbewertung und des Venous Clinical Severity Score (VCSS).

In die Studie wurden 144 Patienten eingeschlossen und insgesamt 162 varikös veränderte Stammvenen mittels Laserablation behandelt (VSM $n=76$, VSP $n=65, K R n=25$ ). Einschlusskriterien waren eine behandlungsbedürftige Stammvarikose der VSM, VSP oder ein Krossenrezidiv (KR) mit einem Krossenstumpf von mindestens $0,7 \mathrm{~cm}$ Länge. Die Operationen wurden von zwei erfahrenen Phlebochirurgen durchgeführt.

Präoperativ wurde der Schweregrad der Varikose anhand des klinischen Befundes gemäß der CEAP Klassifikation und des Venous Clinical Severity Score (VCSS) bestimmt ( $\triangleright$ Tab. 1$)$. Weiterhin wurde präoperativ duplexsonographisch der Venendurchmesser der VSM bei $3 \mathrm{~cm}$ und $10 \mathrm{~cm}$ ermittelt ( $>$ Tab. 2) Bei der VSP wurde der Durchmesser bei $3 \mathrm{~cm}$ gemessen ( $\triangleright$ Tab. 2). In der Rezi-

> Tab. 1 Verteilung der CVI präoperativ

\begin{tabular}{|l|l|l|l|}
\hline & VSM (n= 76) & VSP (n= 65) & Rezidiv (n=25) \\
\hline C2 & $20(26,3)$ & $15(23)$ & $7(28)$ \\
\hline C3 & $45(59,2)$ & $37(56,9)$ & $15(60)$ \\
\hline C4 & $6(7,9)$ & $10(15,4)$ & $2(8)$ \\
\hline C5 & $1(1,3)$ & $2(3)$ & $1(4)$ \\
\hline C6 & $2(2,6)$ & $1(1,5)$ & 0 \\
\hline
\end{tabular}


- Tab. 2 Baseline Daten angegeben in Mittelwert und (Standardabweichung). DM= Durchmesser, VSM=Vena saphena magna, VSP=Vena saphena parva, KS= Krossenstumpf.

\begin{tabular}{|l|l|l|l|l|l|}
\hline & DM $\mathbf{1}(\mathbf{m m})$ & DM $\mathbf{2}(\mathbf{m m})$ & BMI & Alter & Venenlänge (cm) \\
\hline VSM & $7,23(2,28)$ & $6,34(1,89)$ & $27,54(15,59)$ & $55(13)$ & $44,01(9,62)$ \\
\hline VSP & & $27,73(5,36)$ & $60(12)$ & $17,31(4,97)$ \\
\hline KS & $5,93(1,1)$ & $27,95(5,15)$ & $57(10)$ & $17,3(6,63)$ \\
\hline
\end{tabular}

- Tab. 3 Operationsdaten angegeben in Mittelwert und (Standardabweichung). TLA=Tumeszenz-Lokalanästhesie, NMH= Niedermolekulares Heparin.

\begin{tabular}{|l|l|l|l|l|l|}
\hline & TLA (ml) & Laserenergie (J) & NMH (Tage) & Spez. Energie (J/cm) & Spez. TLA (ml/cm) \\
\hline VSM & 257,22(11,46) & $2481,47(791,79)$ & $7,33(3,61)$ & $56,41(12,75)$ & $5,98(2,25)$ \\
\hline VSP & $123,12(50,01)$ & $787,88(305,58)$ & $6,78(4,17)$ & $45,29(11,97)$ & $7,67(4,15)$ \\
\hline KS & & $301,38(166,92)$ & $6,36(3,6)$ & $168(53,15)$ & \\
\hline
\end{tabular}

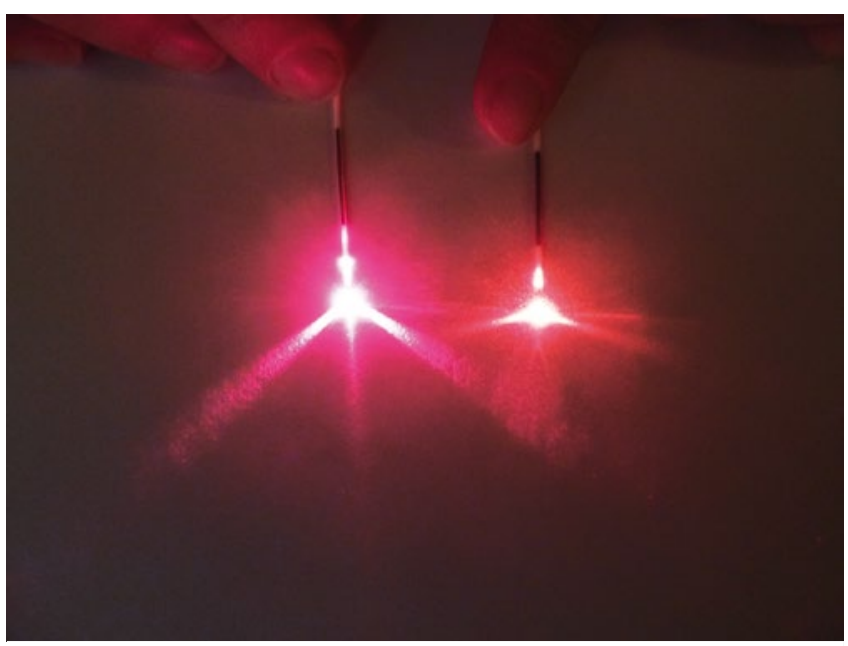

Abb. 1 Diese Abbildung verdeutlicht die unterschiedlichen Abstrahlwinkel der Lasersonden. Rechts die ETQ $360^{\circ}$ FUSED Fiber mit einem Abstrahlwinkel von $60^{\circ}$, links die herkömmliche Lasersonde mit einem Abstrahlwinkel von $80^{\circ}$. Die Interaktion zwischen Gewebe und der Sonde findet durch den geringeren Abstrahlwinkel an der vorderen Spitze der Sonde statt. So sollten eine Karbonisierung der Sonde sowie Verklebungen mit dem Gewebe verringert werden.

divgruppe wurde der maximale Durchmesser des Krossenstumpfs gemessen ( $>$ Tab. 2).

Für die Behandlung der VSM und von KR wurde eine Faser mit einem Faserkerndurchmesser von $600 \mu \mathrm{m}$ bei einem Spitzendurchmesser von 1,8 mm und mit 8 Watt Behandlungsenergie eingesetzt. Zur Behandlung der VSP wurde eine Sonde mit einem Faserkerndurchmesser von $400 \mu \mathrm{m}$ bei einem Spitzendurchmesser von 1,3 mm und 6 Watt Behandlungsenergie gewählt. Die Glasspitze ist jeweils mit der Faser verschweißt und hat den gleichen Durchmesser wie die Faser selbst.
Der Venenzugang erfolgte bei der $400 \mu \mathrm{m}$ Sonde über eine $16 \mathrm{G}$ Venenverweilkanüle bzw. 14G Venenverweilkanüle bei der $600 \mu \mathrm{m}$ Sonde. Eine Schleuse wurde für den Venenzugang nicht benötigt.

Alle Laserbehandlungen der VSM und VSP wurden mit Tumeszenzlokalanästhesie (TLA) durchgeführt. Die Behandlung von KR erfolgte in $88 \%$ in Narkose ohne TLA, 12 \% der KR Eingriffe wurden in TLA durchgeführt.

Bei der Operation wurde eine endovenöse Krossektomie durch mündungsnahe Platzierung der Laserfaser und Mitbehandlung der Vena saphena accessoria anterior bzw. Vena saphena accessoria posterior (falls eine Punktion aufgrund des Gefäßdurchmessers möglich war) angestrebt. Zusätzlich zur endovenösen thermischen Behandlung wurde in 89,8 \% der Fälle eine Schaumverödung der Seitenastvarikose durchgeführt. Bei 25,3\% der Patienten erfolgte eine Miniphlebektomie.

Postoperativ erfolgte in allen Kohorten eine Thromboseprophylaxe mit niedermolekularem Heparin (NMH) über 6 Tage. 6 Patienten hatten bereits präoperativ eine orale Antikoagulation, diese wurde fortgeführt. Lagen Risikofaktoren für eine tiefe Beinvenenthrombose (TVT) vor, wie z. B. TVT in der Anamnese, Östrogeneinnahme oder eine bekannte Thrombophilie, erfolgte eine prolongierte Thromboseprophylaxe über 10-30 Tage. 17 Patienten waren hiervon betroffen.

Klinische und duplexsonographische Verlaufskontrollen erfolgten nach 10-14 Tagen (Visite 2) und nach 3 Monaten (Visite 3). Alle Patienten wurden nach 10-14 Tagen nachkontrolliert (Visite 2). Zu Visite 3 sind 95,2\% der Patienten erschienen. Anhand der Anamnese wurde die Patientenzufriedenheit mithilfe einer Schulnotenskala von 1-6 beurteilt. Außerdem wurden die Dauer des Analgetika-Einsatzes sowie die Arbeitsunfähigkeit in Tagen dokumentiert. In der klinischen Untersuchung wurde der VCSS, das Auftreten von Ecchymosen, Hyperpigmentierungen oder neurologischen Defiziten (z. B. Parästhesie/Dysästhesie) erfasst. Die statistische Auswertung des VCSS erfolgte mit dem CHI Quadrat Test nach Kruskal-Wallis. 
Duplexsonographisch wurde der Behandlungserfolg, der Abstand des proximalen Anteils der okkludierten Vene bis zur sapheno-femoralen Junktion beurteilt. Außerdem wurde das Auftreten von einer PATE „post ablation thrombus extension“ und TVT ausgeschlossen. Die Einteilung der PATE erfolgt in Anlehnung an PASTE und EHIT [7]:

- PATE 0: Ausdehnung des Thrombus bis zur tiefen Vene (= planer Verschluss = gewünschter Therapieerfolg)

- PATE I: Ausdehnung des Thrombus wenige Millimeter in die tiefe Vene hinein mit Einengung des Lumens bis zu 25\%

- PATE II: Ausdehnung in die tiefe Vene hinein mit Einengung des Lumens bis maximal $50 \%$

- PATE III: Einengung der tiefen Vene $>50 \%$

- PATE IV: Vollständiger kurzstreckiger Verschluss der tiefen Vene

Eine Einverständniserklärung zur Datenerhebung aller in die Studie eingeschlossenen Patienten liegt vor.

\section{Ergebnisse}

\section{VSM Gruppe}

Alle thermisch behandelten VSM ( $n=76)$ waren bei Visite 2 und Visite 3 verschlossen. Hyperpigmentierungen zeigten sich bis Visite 3 bei 9,6\%. Ecchymosen im behandelten Areal wurden bei $11,8 \%$ in Visite 2 diagnostiziert, diese bildeten sich in allen Fällen bis Visite 3 zurück. Sensibilitätsstörungen traten bei 6,6\% auf und reduzierten sich bis zu Visite 3 auf 1,4\%. Eine PATE Grad II wurde in einem Fall $(1,4 \%$ ) bei Visite 2 festgestellt, nach einer therapeutischen Behandlung mit NMH für 20 Tage zeigte sich ein Normalbefund. Eine TVT trat in keinem Fall auf. Die Arbeitsunfähigkeit betrug im Mittelwert 1,59 Tage, die Dauer der Einnahme von Schmerzmitteln postoperativ 0,37 Tage. Der VCSS verbesserte sich im Mittelwert signifikant $(p<0,05)$ von 6,22 präoperativ auf 2,07 nach 3 Monaten.

\section{VSP Gruppe}

Alle behandelten VSP $(n=65)$ waren bei Visite 2 und 3 verschlossen. Ecchymosen bestanden in 2 Fällen bei Visite 2, diese bildeten sich bis Visite 3 zurück. Hyperpigmentierungen traten bis Visite 3 in 2 Fällen auf $(3,1 \%)$. Sensibilitätsstörungen bestanden in Visite 2 in 5 Fällen (7,69\%) und reduzierten sich auf 4 Patienten $(6,15 \%)$ bei Visite 3. Eine PATE oder eine TVT wurde in keinem Fall festgestellt. Die Arbeitsunfähigkeit betrug im Mittelwert 0,97 Tage, die Einnahme von Schmerzmittel 0,45 Tage. Der VCSS verbesserte sich im Mittelwert signifikant $(p<0,05)$ von 6,54 präoperativ auf 1,41 nach 3 Monaten.

\section{Krossenrezidiv Gruppe}

Ein Verschluss des Krossenstumpfs zeigte sich in Visite 2 bei $96 \%$. In einem Fall (4\%) kam es zu einem Teilverschluss mit Reflux. Nach 3 Monaten waren $80 \%$ der Krossenstümpfe verschlossen, in einem Fall (4\%) zeigte sich ein Teilverschluss ohne Reflux, in 3 Fällen (12\%) ein Teilverschluss mit Reflux und in einem Fall (4\%) zeigte sich ein vollständig rekanalisierter Krossenstumpf mit Reflux. Eine Korrelation zum Durchmesser des Stumpfes oder zur Länge des Krossenstumpfs bestand im Falle eines Therapieversagens nicht. Ebenso wenig bestand ein Zusammenhang zwischen der Antikoagulation und der Verschlussrate. Eine TVT trat in keinem Fall auf, eine PATE 2 trat bei einem Patienten auf (4\%). Nach Behandlung mit Rivaroxaban $20 \mathrm{mg}$ pro Tag über 28 Tage bildete sich der Appositionsthrombus vollständig zurück. Die Arbeitsunfähigkeit betrug im Mittelwert 3,16 Tage, die Schmerzmitteleinnahme im Mittelwert 0,64 Tage. Der VCSS verbesserte sich im Mittelwert signifikant $(p<0,05)$ von präoperativ 6,48 auf 1,75 nach 3 Monaten.

\section{Diskussion}

Die Behandlung der VSM mit der ETQ $360^{\circ}$ FUSED Fiber der Firma Endoteq $^{\mathrm{TM}}$ zeigte nach 3 Monaten einen $100 \%$ igen Verschluss bei geringem Nebenwirkungsprofil. Ähnlich gute Ergebnisse zeigte bereits Pannier et al 2011 mit dem Radiallaser 1470 nm der Firma Biolitec $^{\mathrm{TM}}$ an 50 VSM Behandlungen mit einer Verschlussrate von $100 \%$ nach 6 Monaten unter Verwendung einer deutlich höheren Energiedichte von 90,8 J/cm. Schwere Komplikationen wie TVT traten auch in dieser Studie nicht auf, jedoch zeigte sich eine etwas höhere Rate an Sensibilitätsstörungen (6\%). In unserer Studie traten nur 1,4\% Sensibilitätsstörungen nach drei Monaten auf. Dies könnte darauf hinweisen, dass durch die Reduktion der Energie bei Einsatz der ETQ $360^{\circ}$ FUSED Fiber das Auftreten von Nebenwirkungen wie z. B. Sensibilitätsstörungen verringert werden kann. Die Verschlussraten sind mit Fasern höherer Energiedichten vergleichbar.

In einer Metaanalyse von Boersma et al. 2016 [1] zur endovenösen Behandlung der VSP wurde bei der EVLA Methode eine Verschlussrate von $98,5 \%$ bei 2950 Fällen in insgesamt 28 Studien gefunden. Sensibilitätsstörungen wurden bei $4,8 \%$ der Patienten erfragt. Samuel et al. 2013 [6] berichtet bei 53 mit EVLA behandelten VSP von einer Verschlussrate von 96,2\% nach einem Jahr. Wir konnten mit der ETQ $360^{\circ}$ FUSED Fiber ${ }^{\mathrm{TM}}$ nach 3 Monaten eine Verschlussrate von $100 \%$ erreichen. Sensibilitätsstörungen traten bei 6,15\% der Fälle auf. Unter Berücksichtigung des kurzen Zeitintervalls der Nachkontrollen sind unsere Ergebnisse mit denen von Borsma et al. und Samuel et al vergleichbar. Insgesamt konnten wir eine hohe Patientenzufriedenheit erreichen, die Arbeitsunfähigkeit betrug nur wenige Tage.

Die offene Rezidivkrossektomie bei lang belassenem saphenofemoralen Stumpf ist aufgrund von Vernarbungen anspruchsvoll und mit einem erhöhten Operationsrisiko verbunden. Vergleichsstudien zur Behandlung von reinen Krossenrezidiven mit EVLA haben wir bei einer Recherche in Pubmed nicht gefunden. Veröffentlichungen zur Behandlung der Rezidivvarikose mit EVLA beziehen sich auf verbleibende VSM oder VSP Stammvenensegmente [3].

Die Ergebnisse dieser Studie mit einer Verschlussrate der KR von $80 \%$ nach 3 Monaten ohne Auftreten von schweren Komplikationen sind vielversprechend. Die Länge der behandelten Krossenstümpfe lag zwischen $0,8 \mathrm{~cm}$ und 3,5 cm (Mittelwert 1,66 cm). Eine mittlere spezifische Energie von $168 \mathrm{~J} / \mathrm{cm}$ wurde eingesetzt. $80 \%$ der Patienten gaben keine Schmerzen durch die EVLA Behandlung an. Unsere Erfahrungen deuten auf die Sicherheit und Effektivität der EVLA Behandlung von sapheno-femoralen Krossenstümpfen hin.

Voraussetzung der EVLA Behandlung von sapheno-femoralen Krossenstümpfen ist die Erfahrung des Operateurs bei duplexso- 
nographisch kontrollierten Punktionen und Platzierung der Laserfaser im Krossenstumpf.

Vergleiche in der aktuellen Literatur zeigen ähnliche Verschlussraten zwischen EVLA sowie RFA (Radiofrequenzablation). Der Einsatz von RFA ist jedoch im Vergleich zur klassischen Chirurgie sowie EVLA mit einem geringeren periprozeduralen Schmerz sowie mit einem geringeren Analgetikaverbrauch assoziiert [9, 10].

Zusammenfassend kann mit der ETQ $360^{\circ}$ FUSED Fiber der Firma Endoteq ${ }^{\mathrm{TM}}$ ein zuverlässiger Verschluss der VSP und VSM erreicht werden. Dieser ist mit einer niedrigen Rate von schweren Komplikationen wie z. B. TVT assoziiert. Durch Verwendung von $6 \mathrm{Watt}$ und einer spezifischen Energieabgabe von 45,3 J/cm hatten wir uns jedoch vor allem bei Behandlung der VSP eine signifikante Reduktion von Sensibilitätsstörungen erhofft. Die klinische Jahreskontrolle wird Klarheit über die Reversibilität der Sensibilitätsstörungen in den behandelten Segmenten bringen. Ein klarer Vorteil der ETQ $360^{\circ}$ FUSED Fiber gegenüber häufiger eingesetzten Sonden mit einem Abstrahlwinkel von $80^{\circ}$ konnte jedoch nicht festgestellt werden.

Abzuwarten bleibt bei allen Gruppen die Entwicklung der sapheno-femoralen und sapheno-poplitealen Stumpflänge und des damit möglichen erhöhten Rezidivrisikos in der Einjahreskontrolle.

Interessenskonflik

Die Studie wurde durch die Firma Endoteq mitfinanziert.
[1] Boersma D, Kornmann VN, van Eekeren RR, et al. Treatment Modalities for Small Saphenous Vein Insufficiency: Systematic Review and Metaanalysis. J Endovasc Ther 2016; 23 (1): 199-211

[2] Goldman MP, Mauricio M, Rao J. Intravascular 1320-nm laser closure of the great saphenous vein: a 6 - to 12-month follow-up study. Dermatol Surg 2004; 30 (11):1380-1385

[3] Nwaejike N, Srodon PD, Kyriakides C. Endovenous laser ablation for the treatment of recurrent varicose vein disease--a single centre experience. Int J Surg 2010; 8 (4):299-301

[4] Pannier F, Rabe E, Maurins U. First results with a new 1470-nm diode laser for endovenous ablation of incompetent saphenous veins. Phlebology 2009; 24 (1): 26-30

[5] Pannier F, Rabe E, Rits J et al. Endovenous laser ablation of great saphenous veins using a $1470 \mathrm{~nm}$ diode laser and the radial fibre - follow-up after six months. Phlebology 201; 26 (1): 35-9

[6] Samuel N, Carradice D, Wallace T et al. Randomized clinical trial of endovenous laser ablation versus conventional surgery for small saphenous varicose veins. Ann Surg 2013; 257 (3): 419-26

[7] Schäffer N, Weingard I, Schuler L et al. Appositionsthrombus als Komplikation endovenöser Katheterverfahren (Post ablation thrombus extension (PATE)). Phlebologie 2018; 47 (02): 93-101

[8] von Hodenberg E, Zerweck C, Knittel M et al. Endovenous laser ablation of varicose veins with the $1470 \mathrm{~nm}$ diode laser using a radial fiber - 1-year follow-up. Phlebology 2015; 30 (2): 86-90

[9] Nordon IM, Hinchliffe RJ, Brar Ret al. A prospective double-blind randomized controlled trial of radiofrequency versus laser treatment of the great saphenous vein in patients with varicose veins. Ann Surg $2011 ; 254$ (6): 876-8

[10] Hinchliffe RJ, Ubhi J, Beech A et al. A prospective randomised controlled trial of VNUS closure versus surgery for the treatment of recurrent long saphenous varicose veins. Eur J Vasc Endovasc Surg 2006; 31 (2): 212-8 